\title{
A novel bicompartmental mathematical model of glioblastoma multiforme
}

\author{
LEITH HATHOUT $^{1}$, BENJAMIN M. ELLINGSON ${ }^{2,4}$, TIMOTHY CLOUGHESY ${ }^{3,4}$ and WHITNEY B. POPE ${ }^{2}$ \\ ${ }^{1}$ Harvard Medical School, Boston, MA 02115-5750; Departments of ${ }^{2}$ Radiological Sciences and \\ ${ }^{3}$ Neurology, David Geffen School of Medicine at UCLA; ${ }^{4}$ Jonsson Comprehensive \\ Cancer Center, UCLA Medical Center, Los Angeles, CA 90024, USA
}

Received September 12, 2014; Accepted October 24, 2014

DOI: $10.3892 /$ ijo.2014.2741

\begin{abstract}
Glioblastoma multiforme (GBM) is the most common primary CNS neoplasm, and continues to have a dismal prognosis. A widely-used approach to the mathematical modeling of GBM involves utilizing a reaction-diffusion model of cell density as a function of space and time, which accounts for both the infiltrative nature of the tumor using a diffusion term, and the proliferation of tumor cells using a proliferation term. The current paper extends the standard models by incorporating an advection term to account for the so-called 'cell streaming' which is often seen with GBM, where some of the tumor cells seem to stream widely along the white matter pathways. The current paper introduces a bicompartmental GBM model in the form of coupled partial differential equations with a component of dispersive cells. The parameters needed for this model are explored. It is shown that this model can account for the rapid distant dispersal of GBM cells in the CNS, as well as such phenomena as multifocal gliomas with tumor foci distant from the core tumor site. The model suggests a higher percentage of tumor cells below the threshold of MRI images in comparison to the standard model. By incorporating an advection component, the proposed model is able to account for phenomena such as multicentric gliomas and rapid distant dispersion of a small fraction of tumor cells throughout the CNS, features important to the prognosis of GBM, but not easily accounted for by current models.
\end{abstract}

\section{Introduction}

Malignant gliomas represent the most common primary central nervous system tumor type in adults, with cerebral

Correspondence to: Dr Leith Hathout, Harvard Medical School, Vanderbilt Box 263, 107 Avenue Louis Pasteur, Boston, MA 021155750, USA

E-mail: leith_hathout@hms.harvard.edu

\section{Abbreviation: GBM, glioblastoma multiforme}

Key words: cell migration, gliobastoma multiforme, mathematical modeling, differential equations, growth kinetics glioblastoma multiforme (GBM) making up the bulk of these neoplasms (1). Despite significant advances in molecular biology and brain tumor therapy, there has been little improvement in GBM prognosis, with mean survival times of 12-14 months (2-5). This is felt to be due to the highly infiltrative nature of GBM, with motile invading cells extending beyond the surgical margins and radiation therapy fields, almost invariably leading to tumor recurrence and death $(1,6)$. Thus, a better understanding of GBM behavior is needed in order to improve GBM treatment strategies and prognosis. Mathematical modeling of GBM is one approach used to try to gain increased understanding of GBM behavior. Specifically, GBM models seek to understand how a tumor grows and spreads out over time. A particularly important parameter to model is tumor cell density as a function of space and time as this may have important implications for determining appropriate surgical margins and radiation fields.

One approach to the mathematical modeling of GBM has been pioneered by Alvord et al, Tracqui et al, Swanson et al and Murray et al, using a reaction-diffusion model of cell density as a function of space and time, which accounts for both the infiltrative nature of the tumor using a diffusion term, and the proliferation of tumor cells using a proliferation term (7-10).

In words, the equation of the tumor model can be stated as follows: Rate of change of tumor cell density (at a location $\mathrm{x}$ ) = Net invasion (diffusion) of tumor cells + net proliferation of tumor cells.

In mathematical terms, this may be restated as (9):

$$
\frac{\partial c(t, x)}{d t}=\nabla \cdot(D(x) \nabla c)+\varrho c\left(1-\frac{c}{K}\right)
$$

where the various terms are defined as follows: $c(t, x)$ is the tumor cell density, in terms of cells $/ \mathrm{mm}^{3}$, which is a function of position $x$ and time $t$.

$D(x)$ is the diffusion term, in $\mathrm{mm}^{2}$ /day, which models local tumor invasion of tumor cells

$$
\varrho c\left(1-\frac{c}{K}\right)
$$

is a logistic tumor growth term, where $\varrho$ is the tumor proliferation rate in units of (/day), governed by a tissue tumor carrying capacity $K$, in units of cells $/ \mathrm{mm}^{3}$. 
With a constant diffusion term, and in one spatial dimension, we get the classical Fisher-Kolmogoroff reaction diffusion equation successfully used by previous investigators to model many aspects of GBM growth dynamics $(7,8,10)$ :

$$
\frac{\partial c}{d t}=D \frac{\partial^{2} c}{d x^{2}}+\varrho c\left(1-\frac{c}{K}\right)
$$

This model has also been successfully extended by Wang et al to relate prognosis to the growth kinetic parameters characterizing diffusion and proliferation and by Rockne et al to model the response of GBM to radiation $(9,11)$.

The Fisher-Kolmogoroff equation and GBM models derived from it are an example of the general class of conservation of matter equations with a source term,

$$
\frac{\partial c(t, x)}{d t}+\nabla \cdot J-f=0
$$

where $J$ represents the flux function and $f$ represents the source term, and where $J$ is due to diffusion alone i.e., $J=-D \nabla c$ (12).

There are, however, several lines of evidence which indicate that a flux function consisting only of a diffusion term may be incomplete for the purposes of modeling the behavior of GBM. One of these is a rapid dispersal of tumor cells throughout the CNS in a manner which cannot be accounted for by diffusion, which is essentially a local phenomenon (13-15). For example, Silbergeld and Chicoine have demonstrated that within 7 days of local implantation of human GBM cells into a rat brain, tumor cells can be identified throughout the central nervous system (1). It can be shown (see below) that with diffusion alone, it would take significantly longer for this to occur. Burgoyne et al noted the same phenomenon, where a locally dense tumor remains at the site of implantation, but some cells migrate away quickly from the main tumor bulk to disperse widely in the CNS (16). In other words, this evidence suggests that tumor cell migration cannot be thought of solely as a process of random motion. Instead, there must be a component of tumor cell migration that is an active, non-random process.

The purpose of the current paper is to extend the above reaction diffusion models by incorporating an advection term to account for the so-called 'cell streaming' which is often seen with glioblastoma multiforme, where some of the tumor cells seem to stream widely along the white matter pathways. This occurs only with a small proportion of the tumor cells, which Burgoyne et al term 'dispersive cells' (16). However, this distant dispersal of tumor cells seems to be a crucial part of the poor prognosis of GBMs. It leads not only to tumor recurrence beyond the therapy field, but can also lead to a phenomenon such as multifocal-multicentric GBM. This suggests that GBMs need an additional advection component to adequately model their behavior.

The current paper introduces a bicompartmental GBM model in the form of coupled partial differential equations with a component of dispersive cells. It is shown that this model can account for the rapid distant dispersal of GBM cells in the CNS, as well as such phenomena as multifocal gliomas with tumor foci distant from the core tumor site, neither of which is easily explained by the current reaction diffusion model.

\section{Model}

To account for a component of 'dispersive' cells, a model consisting of coupled partial differential equations is employed. Given an initial cell population defined by $c(0, x)$, a small fraction of the cells, $f$, are considered to be dispersive cells with an advection velocity $v$ through the brain. Thus, the cells are considered as two cell populations, $c_{1}$ and $c_{2}$, with a majority of the cells, fraction (1- $f$ ), being in the first population, governed by the usual diffusion-reaction equation, and a much smaller fraction, $f$, being in the second motile population, governed by an advection-diffusion-reaction equation. This system can be expressed as follows:

$$
\begin{aligned}
& \frac{\partial c_{1}}{d t}=D \frac{\partial^{2} c_{1}}{d x^{2}}+\varrho c_{1}\left(1-\frac{c}{K}\right) \\
& \frac{\partial c_{2}}{d t}=D \frac{\partial^{2} c_{2}}{d x^{2}}-v \frac{\partial c_{2}}{d x}+\varrho c_{2}\left(1-\frac{c}{K}\right),
\end{aligned}
$$

Equation [2]

where $c_{1}=(1-f)^{*} c, c_{2}=f^{*} c$, and hence, $c_{1}+c_{2}=c$.

Here, $v$ is the velocity of advection, and $D$ and $\varrho$ are the diffusion and proliferation coefficients.

The initial and boundary conditions are as conventionally employed in the reaction diffusion models of Swanson and Rockne et al (11). At each time point, the cell density $c(x, t)$ for the tumor would be the sum of the two populations, $c=c_{1}+c_{2}$. Specifically, we use $c(0, x)=0.8 K^{*} e^{-0.25 x^{2}}$. The carrying capacity for the tissue, $K$, can be considered a cell density of $10^{5}$ cells $/ \mathrm{mm}^{3}$ (17). The solution domain is $\mathrm{L}=200 \mathrm{~mm}$, and we use the standard zero-flux boundary conditions

$$
\frac{\partial c}{d x}=0
$$

at $x=0$ and at $x=\mathrm{L}(18)$.

For the purposes of calculation, values similar to those used by Rockne et al for $D\left(0.4 \mathrm{~mm}^{2} /\right.$ day $)$ and $\varrho(0.04 /$ day $)$ are employed, serving as average or representative values from a broad range of GBMs (18).

Additionally, $f$, the fraction of cells in the dispersive compartment needs to be specified. From the work of Silbergeld and Chicoine injecting human GBM cells into rat brain, it is clear that this is quite small, since most injected cells remain and grow in a fixed tumor bulk (1). In this paper, $f$ is set to 0.001 , but this is a model parameter which will need to be empirically determined in further studies.

Also, an advection velocity $v$ needs to be input for the small fraction of dispersive cells in the $c_{2}$ compartment. A maximum value for this velocity can be approximated from the equation $v=l / \tau$, where $l$ is the length over which cells migrated and $\tau$ is the time which this took. This approach gives an order of magnitude approximation, but ignores the motion of the cells secondary to diffusion. A more rigorous derivation is possible using a simplified model so as to be mathematically tractable, but which incorporates the effects of both $D$ and $v$ into the mean transit time $\tau$ for a diffusion process.

Thus, to get an approximate sense, we can begin the derivation by examining the transit time $\tau$ in a steady state situation. 
In a one dimensional linear model as above, the average transit time $\tau$ from $x=0$ to $x=l$ represents the time it would take the total amount of cells $N$ to leave the region given a flux of $J$, which, in turn, represents the amount of cells passing a point per unit time. In other words, $\tau=N / J$.

This approach, for example, can be used to calculate the mean transit time $\tau$ for a simplified steady-state system where the flux $J$ is due to diffusion alone. This means that:

$$
J=-D \frac{\partial c}{d x} .
$$

In looking at the rat GBM model of Silbergeld and Chicoine, we postulate $c_{0}$ cells implanted at $x=0$, and in steady state, we have the equation:

$$
0=D \frac{\partial^{2} c}{d x^{2}},
$$

in $(0, l)$, with $c(0)=c_{0}, c(l)=0$.

This has a solution:

$$
c(x)=c_{0}\left(1-\frac{x}{l}\right) .
$$

The flux is thus

$$
J=-D \frac{\partial c}{d x}=D \frac{c_{0}}{l} .
$$

The number of cells in the domain is

$$
N=\int_{0}^{1} c(x) d x=\frac{1}{2} l c_{0} .
$$

Thus, the mean transit time is

$$
\tau=N / J=\frac{\frac{1}{2} l c_{0}}{D \frac{c_{0}}{l}}=\frac{1}{2} \frac{l^{2}}{D}
$$

Using the same approach with a simplified advection diffusion model in steady state, it is also possible to derive some estimate of $\tau$ in terms of $v$ and $D$, and use that to get some estimates of $v$ if $\tau$ is known (12). Ignoring the proliferation term is at least partially justified by noting that in the solution of the reaction-diffusion model above, the proliferation term does not significantly impact the cell concentration profile in the early part of the system's evolution, i.e., the time during which cells stream widely away from the tumor in the Silbergeld and Chicoine rat model, for example.

In steady state, the advection-diffusion model reduces to:

$$
0=D \frac{\partial^{2} c}{d x^{2}}-v \frac{\partial c}{d x},
$$

in $(0, l)$, with $c(0)=c_{0}, c(l)=0$.

The general solution of this second order differential equation is

$$
c(x)=A+B\left(\frac{\mathrm{D}}{\mathrm{v}}\right) \mathrm{e}^{v x / D},
$$

where $A$ and $B$ are arbitrary constants.
The boundary conditions lead to the particular solution

$$
c(x)=c_{0} \frac{\mathrm{e}^{v l / D}-\mathrm{e}^{v x / D}}{\mathrm{e}^{v / D}-1} .
$$

To get $N$, the total number of cells in $(0, l)$, we need to integrate $c(x)$ across the whole interval:

which gives

$$
N=\int_{0}^{1} c(x) d x
$$

$$
N=c_{0} \frac{l e^{v / D}-(D / v)\left(e^{v / / D}-1\right)}{e^{v l / D}-1}
$$

The flux $J$ is given by

$$
J=v c-D \frac{d c}{d x},
$$

which from the above evaluates to

$$
J=v c_{0} \frac{e^{v l / D}}{e^{v l / D}-1} .
$$

Finally,

$$
\tau=\frac{N}{J}=\frac{l}{v}-\frac{D}{v^{2}}\left(1-e^{v l / D}\right)
$$

This represents the initial approximation of $\tau=l / v$, but now modified to take account of the fact that some of the cell motion is due to diffusion.

It is interesting to note that as $v$ approaches 0 , the limit of $\tau$ in the expression above is $\tau=l^{2} / 2 D$, the same as when the flux term is due to diffusion alone, as in equation (3).

This can be seen by expanding the exponential term using a Taylor series approximation:

$$
\tau=\frac{l}{v}-\frac{D}{v^{2}}\left(1-\left(1-\frac{v l}{D}+\frac{v^{2} l^{2}}{2 D^{2}}+f\left(v^{n}\right)\right)\right),
$$

where $f\left(v^{n}\right)$ represents higher order terms in $v$.

Using Equation 4 and having estimates for $D, l$ and $\tau$, it is then possible to calculate $v$.

For the purposes of simulations in this model, an average velocity $v$ of $0.75 \mathrm{~mm} /$ day is used (see Discussion).

\section{Results}

The standard reaction-diffusion model provides an evolution of the tumor cell density with time, where cell density gradually increases toward the carrying capacity $K$ and where the tumor grows in space, with the tumor cell-density curve moves to the right (Fig. 1).

The bicompartmental reaction-diffusion-advection model of Equation 2 also gives a time evolution of tumor cell density, broadly similar to the standard model, but with some significant differences (Fig. 2).

It is noted that in the bicompartmental model, there is significantly more cell streaming, with a low concentration of cells moving away from the tumor bulk even at early time points. This can be seen by comparing the extent of the tail along the $\mathrm{x}$ axis at comparable time points for both sets of 

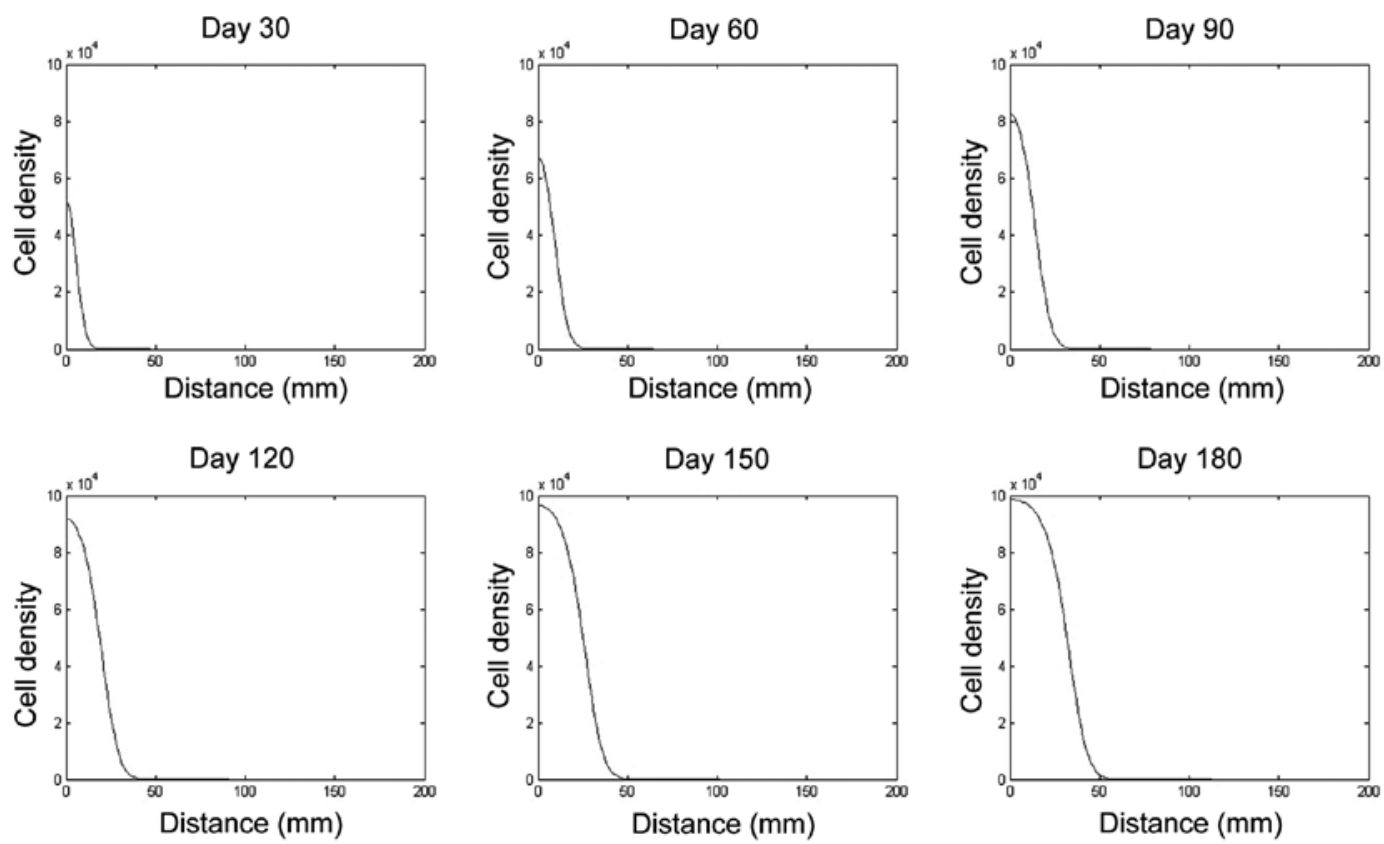

Figure 1. The time evolution of the cell density profile as given by the standard reaction-diffusion model of Equation 1 , with $c(0, x)=0.8 K^{*} e^{-0.25 x^{2}}$, $K=10^{5}$ cells $/ \mathrm{mm}^{3}, D\left(0.4 \mathrm{~mm}^{2} /\right.$ day $)$ and $\varrho(0.04 /$ day $)$.
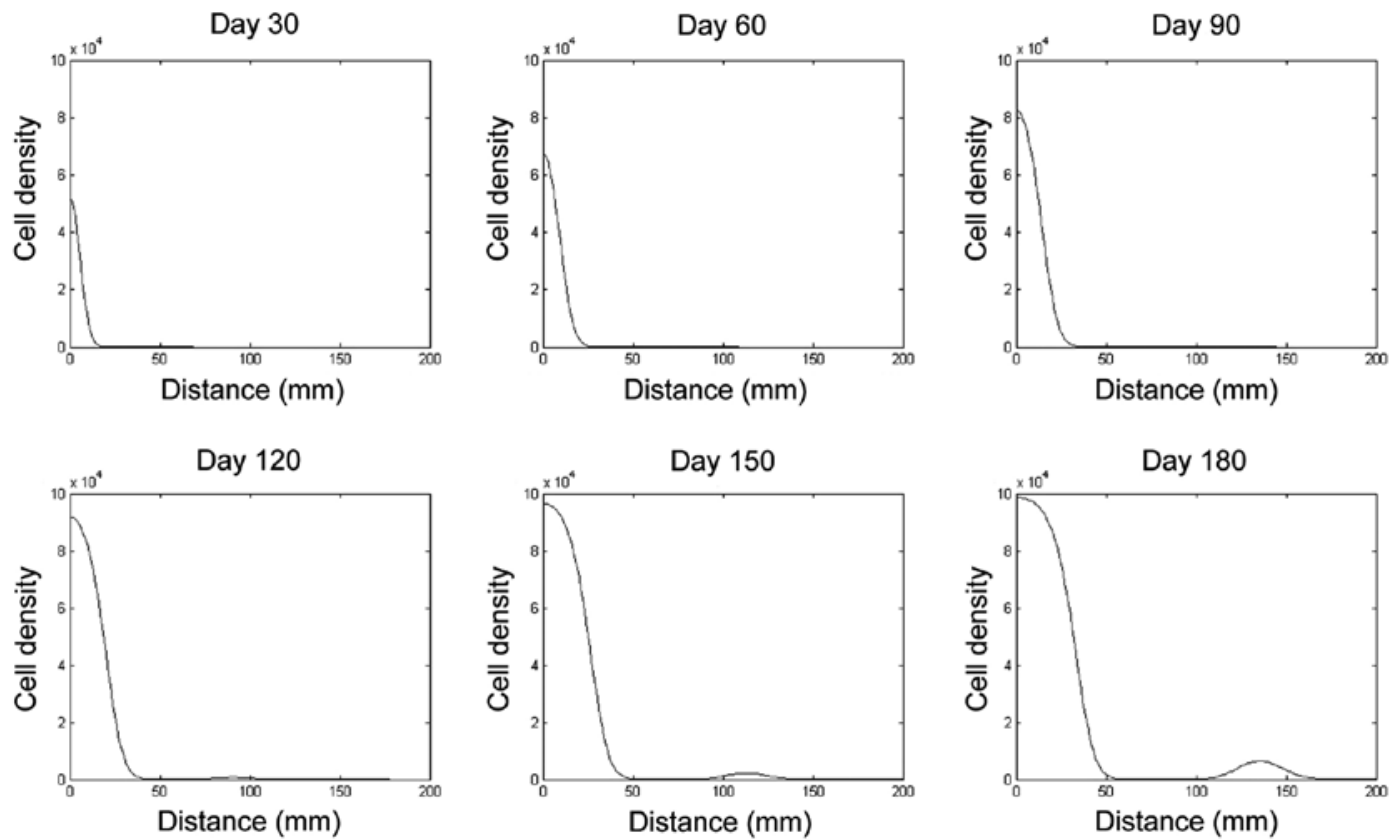

Figure 2. The time evolution of the cell density profile as given by the system of equations representing the reaction-diffusion-advection model of Equation 2 , with the same initial and boundary conditions as the standard model, and with $f=0.001, v=0.75 \mathrm{~mm} / \mathrm{day}$.

curves in Figs. 1 and 2. This can sometimes be seen on imaging (Figs. 3 and 4), but is typically below the threshold of imaging detection (see Discussion).

It is noted that both the conventional model and the bicompartmental model show the expected theoretical linear growth of tumor, with a constant increase in tumor radius with time. According to the Fisher-Kolmogoroff equation, with large time $t$, the velocity of the tumor can be approximated by $v=2 \sqrt{D \varrho}(9,10)$. For the model values of $D\left(0.4 \mathrm{~mm}^{2} /\right.$ day $)$ and $\varrho(0.04 / \mathrm{day})$, this would correspond to an increase of $0.253 \mathrm{~mm} /$ day in tumor radius. This is seen in a plot of tumor radius with time, where a least-squares linear fit shows a velocity of $0.258 \mathrm{~mm} /$ day (Fig. 5).

\section{Discussion}

This paper presents a preliminary 'proof of concept' investigation into extending the standard reaction-diffusion model of 


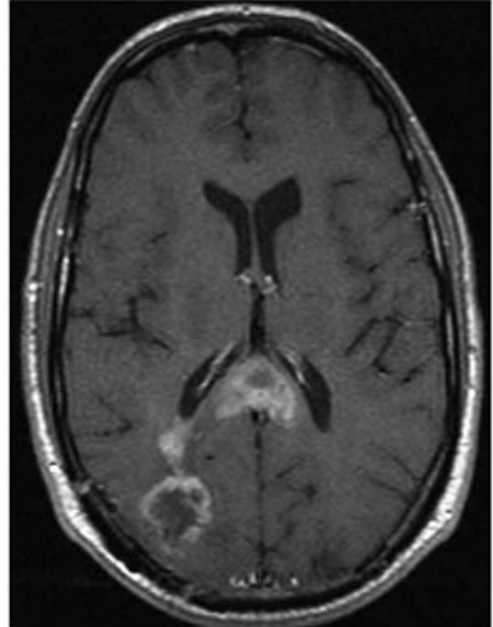

Figure 3. T1 weighted post-contrast axial image shows enhancing tumo cells streaming away from a right occipital surgical resection cavity where the main tumor was debulked, extending the tumor into the splenium of the corpus callosum
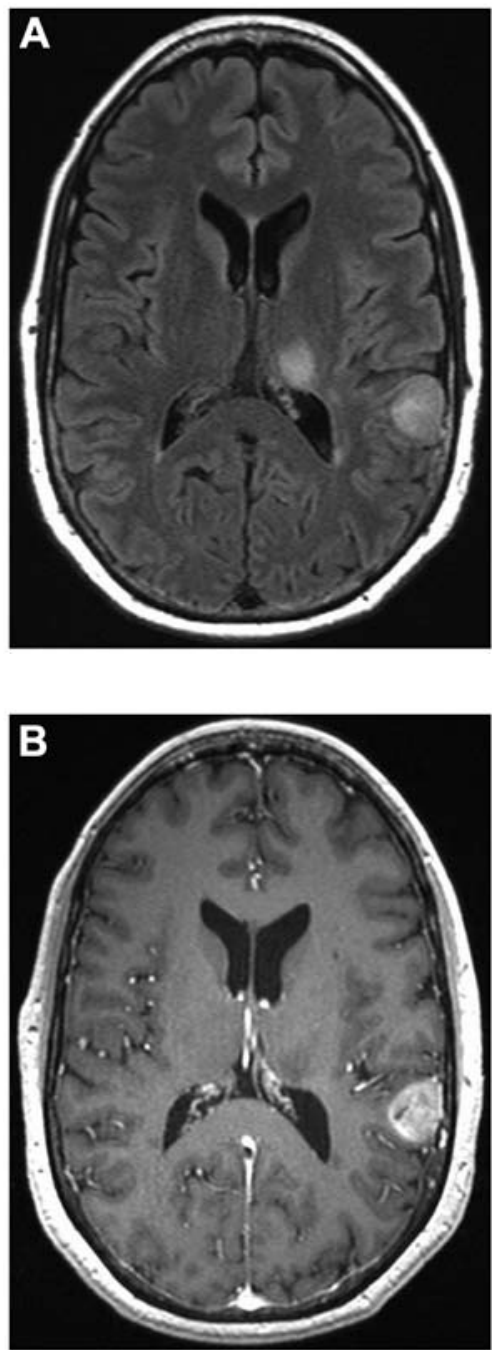

Figure 4. (A) Axial FLAIR image showing a left temporoparietal tumor focus with a distant satellite focus in the left thalamus. (B) T1 weighted postcontrast axial image shows enhancement of the left temporoparietal focus, but a left thalamic focus below the cell density for enhancement, similar to the cell-density curves of Fig. 2.

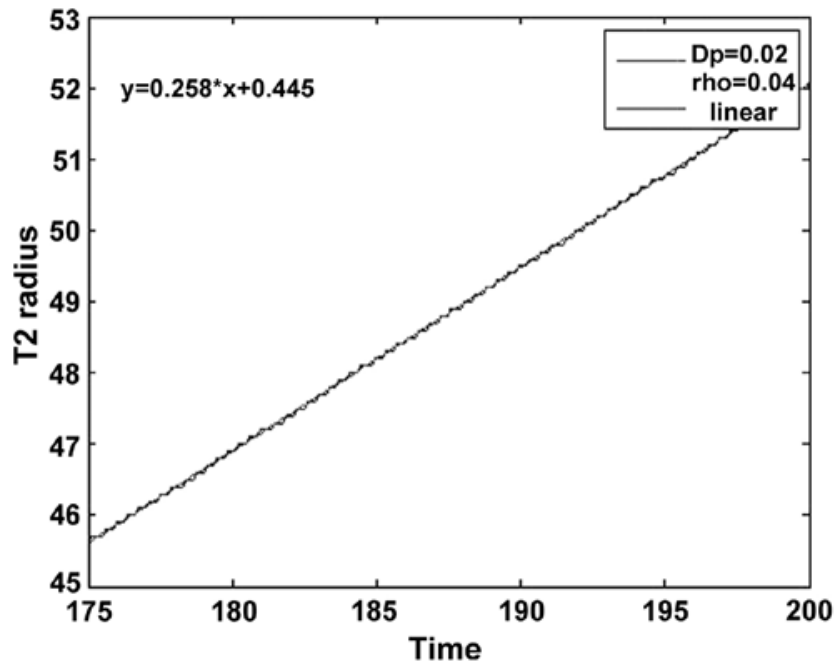

Figure 5. The growth of tumor radius with time, with a superimposed linear least-squares fit, which shows a linear tumor growth with a velocity of $0.258 \mathrm{~mm} /$ day, closely matching the theoretical prediction of $0.253 \mathrm{~mm} / \mathrm{day}$.

GBM to include an advection component to represent distant cell streaming or what Burgoyne et al have termed the dispersive cell fraction (16). Although this fraction represents only a small proportion of the tumor cells, it appears to have several significant correlates in the behavior of GBMs. The most important of these is a rapid spread of tumor cells throughout the CNS. For example, as mentioned above, Silbergeld and Chicoine have demonstrated that within 7 days of local implantation of human GBM cells into a rat brain, tumor cells can be identified throughout the central nervous system $(1,14,15)$. Although a locally dense tumor remains at the site of implantation, some cells stream away quickly from the main tumor bulk. This rapid, distant dispersal of only a small number of cells away from the main tumor cannot be accounted for by diffusion, which is both a local and a bulk phenomenon. Furthermore, using mathematically reasonable estimates of measured D (e.g., $0.4 \mathrm{~mm}^{2} /$ day, as used here), and assuming a dimension of only $1 \mathrm{~cm}$ for the rat brain, Equation 3 shows that with diffusion alone, it would take about 128 days for tumor cells to diffuse through even this limited neuroaxis - much longer than what is actually observed.

This cell streaming and distant dispersal seems to be a crucial part of the poor prognosis of GBMs. Overall, the notion is that while current treatments are focused on tumor control within the gross tumor bed and closely adjacent areas of the brain, it is now clear that malignant glioma cells have likely already widely invaded much of the brain prior to diagnosis and treatment (19-21). Thus, as noted by Burgoyne et al, low grade gliomas are locally infiltrative, but 'this contrasts with the extensive infiltration of GBM tumors, where cells disperse to distal sites throughout the entire brain parenchyma' (16).

This is poignantly seen in the old surgical series by neurosurgeons who, in the face of the poor prognosis of GBM, performed total hemispherectomies in glioma patients where they felt certain that the tumor was macroscopically confined to a single hemisphere. In all cases, histologic examination revealed a diffuse spread of some tumor cells in the CNS, and many patients died of gross tumor recurrence in the contra- 

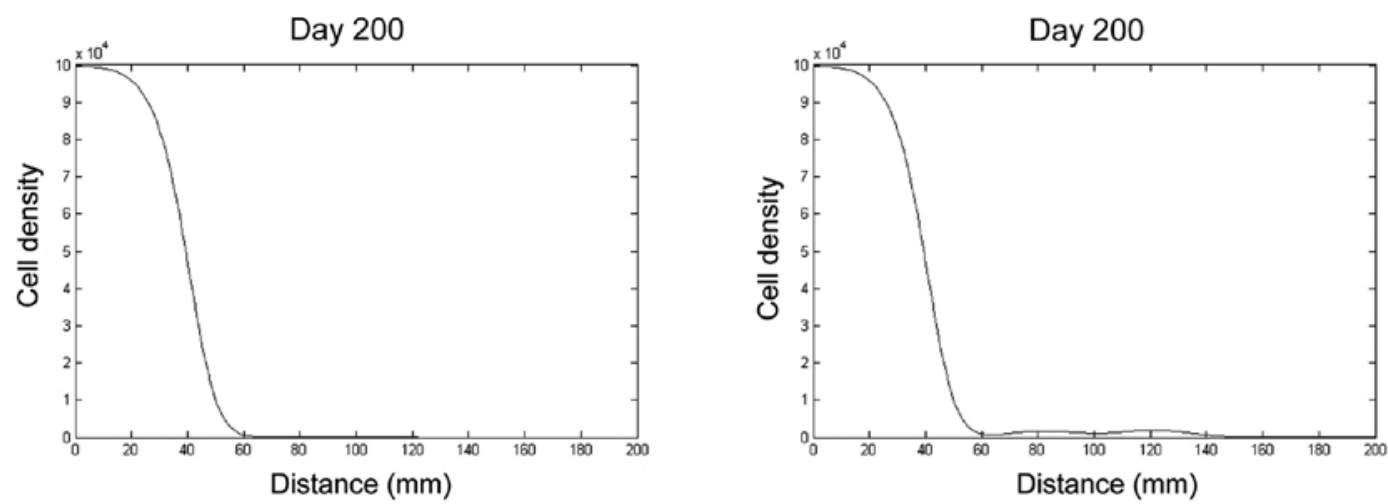

Figure 6. (A) (on the left) shows the output of the standard model at 200 days. (B) (on the right) shows a tricompartment model with two compartments showing advection, with advection velocities of 0.4 and $0.6 \mathrm{~mm} / \mathrm{day}$, and a proliferation rate set at 0.75 of the standard model value. The dispersive cell fraction, $f$, is set at 0.0005 for each compartment, comparable to the $f$ of 0.001 used in earlier simulations.

lateral hemisphere $(4,22,23)$. This propensity for distant tumor migration in GBM was recognized early on, and attributed to phenomenon such as perineuronal satellitosis and intrafasicular tracking $(24,25)$. Various studies have also documented this rapid dispersal of some GBM cells in the CNS on a microscopic basis. Burger et al have noted that with migration along fiber tracts, individual tumor cells are routinely demonstrated up to $6 \mathrm{~cm}$ from the main tumor bulk (19). Giese et al also note that individual tumor cells are detectable several centimeters away from the core lesion, and attribute this to genetic mechanisms that increase cell motility (23).

The current paper introduces a bicompartmental GBM model in the form of coupled partial differential equations with a component of dispersive cells. It retains the excellent predictive capacity of the traditional model, and the ability to incorporate individual patient tumor kinetics, but also accounts for the phenomenon of distant cell migration as a 'gentle' diffuse infiltration distant from the main tumor. As can be seen in the results section (Fig. 2), over time, this cell migration is able to set up distant separate tumor foci which will eventually be visible on imaging (Fig. 4). This result is significant for a variety of reasons. It has the potential to explain the observation of Giese et al of distant tumor sites arising even in the face of local tumor control at the primary site (26).

More generally, if some of the migrated clonogens grow, this model has the potential to explain the phenomenon of multicentric/multifocal glioma without resorting to the notion of separate metachronous or synchronous tumors. As can be seen in Fig. 2, the distant tumor focus will be connected to the main tumor bed by 'invisible' tumor bridges, which are below the threshold of imaging detection. This is in concordance with the assessment of Giese et al that 'many of these tumors may not be truly multifocal but rather may reflect manifestations of more rapidly proliferating foci within a larger area of microscopically invaded brain' (23). The occurrence of such multifocal lesions has been reported in up to $10 \%$ of cases of GBM at initial presentation in older studies (27-29). The more recent study of Patil et al places the incidence at $12.8 \%$ (30). However, the incidence increases significantly in later stages of disease (21). This would be fully in keeping with the predictions of our model, where, with time, more of the distant migrated cells will be able to grow into visible tumor foci under the influence of the proliferation term.

An important point to note is that the current model also accounts for the notion that much of the tumor spread secondary to advection is below the threshold of imaging. In published applications of the reaction-diffusion model, threshold values of cell density are set up to represent detectable tumor on $\mathrm{T} 1$ post-contrast images and on T2 images. The values used for these thresholds have been in the range of $0.8 K$ (where $K$ is the tissue carrying capacity) for T1 post-contrast images and $0.16 K$ for $\mathrm{T} 2$ weighted images (31-33).

Using these thresholds, it is interesting to compare the spread of tumor in the brain which is below the threshold of imaging detection. Numerical simulation shows that at 50 days, the $\mathrm{T} 2$ radius of the tumor is $1.2 \mathrm{~cm}$. Using the conventional model, cell density falls to 0 at $3.2 \mathrm{~cm}$. This is within the typical surgical and radiation therapy margins used in current practice. The bicompartmental model shows significantly more subthreshold tumor spread, with tumor falling to 0 at $6 \mathrm{~cm}$. This can be seen in the longer right hand tail of the cell density curves when comparing Figs. 1 and 2. In fact, at 50 days, $0.15 \%$ of all tumor cells are located between $3.2 \mathrm{~cm}$, where the standard model goes to 0 cell density, and $6 \mathrm{~cm}$. These tumor cells, outside the traditional surgical and radiation therapy margins, are quite significant for the poor prognosis of GBM, in that they provide a source of cells for the tumor recurrence that is typically observed beyond the treatment margins.

More generally, the bicompartmental model predicts more cells beyond the T2 imaging threshold of MRI, and that this proportion increases with time. For example, at 100 days, using the conventional reaction diffusion model, $4.3 \%$ of tumor cells are below the T2 threshold. Using the proposed model presented here, that fraction is $4.8 \%$. At 150 days, however, the numbers are $2.8 \%$ for the conventional model, and $5.1 \%$ for our model, and at 180 days, the fractions are 2.2 vs. $8 \%$, respectively, showing an increasing amount of subthreshold infiltration in the brain with the bicompartmental model.

These findings are significant, since tumor radiation therapy fields, in attempt to avoid the morbidity of whole-brain radiation, are now targeted, with a typical target volume extending $2.5 \mathrm{~cm}$ beyond the visible edge of the tumor on T2 (18). Our model shows cell infiltration well beyond the outer margin of 
the radiation field, and would help explain tumor recurrence at or beyond the margins of the therapy field, as is often seen (23). Moreover, the increasing brain infiltration below the threshold of imaging detection (8\% of tumor cells in the bicompartmental model vs. $2.2 \%$ in the standard model at 180 days) is fully consistent with the observations of Giese et al. In their review on the 'cost of migration' of cells in GBM, they note that many patients die of neurologic deterioration without a large post-treatment tumor bulk, precisely because of the diffuse subthreshold infiltration of the brain (23).

Clearly, mathematical models are idealizations of a much more complex underlying biology, but they have utility if they can capture essential features of the phenomenon under study. As noted above, this paper represents the initial development of an advection-reaction-diffusion model. Thus, one of its purposes is to highlight future research directions. Among the most critical of these are accurate estimates for the model parameters. Previous work on the standard reactiondiffusion model has outlined the approach to estimating $D$ and $\varrho(9,10,31)$. Additional parameters needed for this model are also estimates of $f$, the fraction of dispersive cells and $v$, the advection velocity. The molecular mechanisms of cell motility in GBM are an active area of investigation (34). There is, at this time, little definitive evidence regarding appropriate values for $v$. Chicoine and Silbergeld have noted that in vitro, the motility of human glioma cells increases with increasing tumor grade (15). They have noted that time-lapse videomicroscopy recordings of glioma cells revealed a motility rate of $12.5 \mu \mathrm{m} / \mathrm{h}$ with an ameboid form of locomotion $(1,14)$. This correlates to a speed of about $0.3 \mathrm{~mm} /$ day. In referring to their work with human GBM in the rat brain, Murray quotes a minimum speed of $4.8 \mathrm{microns} / \mathrm{h}$ (10), equating to $0.115 \mathrm{~mm} /$ day. We take this as the minimum available estimate of $v$.

An order of magnitude approximation, which provides an upper limit on $v, v_{\max }$, can perhaps also be found in the rat brain model papers which show that 7 days post-implantation of xenografts into the rat forebrain, individual tumor cells can be found throughout the CNS $(1,14)$. Assuming rat brain of $1 \mathrm{~cm}$, this gives $\tau=7$ days and $l=1 \mathrm{~cm}$, and using $D=0.4 \mathrm{~mm}^{2} /$ day, Equation 4 can be numerically solved for $v$. This gives a $v_{\max }$ of $1.39 \mathrm{~mm} /$ day. Thus, we use a $v$ of $0.75 \mathrm{~mm} /$ day in simulations as roughly representative of the mean between the available minimum and maximum estimates. Clearly, however, more detailed work needs to be done to accurately estimate $v$ in the setting of GBM in vivo in the human brain, as well as to try to obtain some estimates of $f$, the fraction of dispersive cells.

Lack of precise knowledge of these values represents a limitation of the current work. A more general limitation, though, has to do with the nature of continuum modeling itself, where only a single speed $v$ can be assigned to the advection component. Clearly, there is likely a range of advection velocities, depending on both tumor cytoarchitecture, the molecular mechanisms of motility, and the pathway motile cell take (intrafasicular and preineuronal). This limitation may be partially circumvented by adding more compartments to the model. Also, it is unclear whether motile cells will proliferate with the same growth rate as cells within an established tumor, aided by tumor growth factors and angiogenesis promoters. A tricompartment model, for example, may have a cell component with a slower advection velocity and one with a faster advection velocity. One such simulation is presented in Fig. 6 . For reasonable model parameters, it shows a diffuse broadbased infiltration of the brain by tumor cells entirely below the threshold of imaging.

It is once again noted that while this is not entirely realistic, it may help achieve the overall purpose of mathematical modeling, which is to capture some essential features of tumor behavior that may help guide therapy or predict prognosis.

The current work suggests that for therapy planning and estimates of patient prognosis, an advection component needs to be incorporated into the standard diffusion-reaction models, and indicates areas where future research is needed.

\section{References}

1. Silbergeld DL and Chicoine MR: Isolation and characterization of human malignant glioma cells from histologically normal brain. J Neurosurg 86: 525-531, 1997.

2. Salcman M: Survival in glioblastoma: historical perspective. Neurosurgery 7: 435-439, 1980.

3. Salford LG, Brun A and Nirfalk S: Ten-year survival among patients with supratentorial astrocytomas grade III and IV. J Neurosurg 69: 506-509, 1988.

4. Lassman AB and Holland EC: Glioblatoma multiforme - pat, present and future. US Oncology Review 1: 109-111, 2005.

5. Stupp R, Mason WP, van den Bent MJ, et al: Radiotherapy plus concomitant and adjuvant temozolomide for glioblastoma. $\mathrm{N}$ Engl J Med 352: 987-996, 2005.

6. Nazzaro JM and Neuwelt EA: The role of surgery in the management of supratentorial intermediate and high-grade astrocytomas in adults. J Neurosurg 73: 331-344, 1990.

7. Harpold HL, Alvord EC Jr and Swanson KR: The evolution of mathematical modeling of glioma proliferation and invasion. $\mathrm{J}$ Neuropathol Exp Neurol 66: 1-9, 2007.

8. Tracqui P, Cruywagen GC, Woodward DE, Bartoo GT, Murray JD and Alvord EC Jr: A mathematical model of glioma growth: the effect of chemotherapy on spatio-temporal growth. Cell Prolif 28: 17-31, 1995.

9. Wang CH, Rockhill JK, Mrugala, et al: Prognostic significance of growth kinetics in newly diagnosed glioblastomas revealed by combining serial imaging with a novel biomathematical model. Cancer Res 69: 9133-9140, 2009.

10. Murray JD: Growth and control of brain tumors. In: Mathematical Biology II: Spatial Models and Biomedical Applications. Vol. 18, 3rd edition. Antman SS, Marsden JE, Sirovich L and Wiggins S (eds). Springer, New York, pp536-613, 2003.

11. Rockne R, Rockhill JK, Mrugala M, et al: Predicting the efficacy of radiotherapy in individual glioblastoma patients in vivo: a mathematical modeling approach. Phys Med Biol 55: 3271-3285, 2010.

12. Britton NF: Essential Mathematical Biology. Springer, New York, 2003.

13. Bernstein JJ, Goldberg WJ and Laws ER Jr: Human malignant astrocytoma xenografts migrate in rat brains: a model for central nervous system cancer research. J Neurosci Res 22: 134-143, 1989.

14. Chicoine MR and Silbergeld DL: Assessment of brain tumor cell motility in vivo and in vitro. J Neurosurg 82: 615-622, 1995.

15. Chicoine MR and Silbergeld DL: The in vitro motility of human gliomas increases with increasing grade of malignancy. Cancer 75: 2904-2909, 1995.

16. Burgoyne AM, Palomo JM, Phillips-Mason PJ, et al: PTPu suppresses glioma cell migration and dispersal. Neuro Oncol 6: 767-778, 2009.

17. Herculano-Houser S and Lent R: Isotropic fractionator: a simple, rapid method for the quantification of total cell and neuron numbers in the brain. J Neurosci 25: 2518-2521, 2005.

18. Rockne R, Alvord EC Jr, Rockhill JK and Swanson KR: A mathematical model for brain tumor response to radiation therapy. J Math Biol 58: 561-578, 2009.

19. Burger PC, Heinz ER, Shibata T, et al: Topographic anatomy and CT correlations in untreated glioblastoma multiforme. J Neurosurg 68: 698-704, 1988.

20. Salazar OM and Rubin P: The spread of glioblastoma multiforme as a determining factor in the radiation treated volume. Int $\mathbf{J}$ Radiat Oncol Biol Phys 1: 627-637, 1976. 
21. Silbergeld DL, Rostomily RC and Alvord EC Jr: The causes of death in patients with glioblastoma is multifactorial: clinical factors and autopsy findings in 117 cases of supratentorial glioblastoma in adults. J Neurooncol 10: 179-185, 1991.

22. Gardner WJ: Residual functioning following hemispherectomy for tumouir and for infantile hemiplegia. Brain 78: 487-502, 1955.

23. Giese A, Bjerkvig R, Berens ME and Westpha M: Cost of migration: invasion of malignant gliomas and implications for treatment. J Clin Oncol 21: 1624-1636, 2003.

24. Scherer HJ: Structural development in gliomas. Am J Cancer 34 333-351, 1938.

25. Scherer HJ: The forms of growth in gliomas and their practical significance. Brain 63 (part 1): 1-35, 1940.

26. Giese A and Westphal M: Treatment of malignant glioma: a problem beyond the margins of resection. J Cancer Res Clin Oncol 127: 217-225, 2001.

27. Barnard RO and Geddes JF: The incidence of multifocal cerebral gliomas. A histologic study of large hemisphere sections. Cancer 60: 1519-1531, 1987.

28. Batzdorf $\mathrm{U}$ and Malamud $\mathrm{N}$ : The problem of multicentric gliomas. Neurosurgery 20: 122-136, 1970.
29. Budka H, Prodeka I, Reisner T, et al: Diagnostic and pathomorphological aspects of glioma multiplicity. Neurosurg Rev 3: 233-241, 1980.

30. Patil CG, Yi A, Elramsisy A, Hu J, et al: Prognosis of patients with multifocal glioblastoma: a case-control study. J Neurosurg 117: 705-711, 2012.

31. Corwin D, Holdsworth C, Rockne RC, et al: Toward patientspecific, biologically optimized radiation therapy plans for the treatment of glioblastoma. PLoS One 8: e79115, 2013.

32. Szeto MD, Chakraborty G, Hadley, et al: Quantitative metrics of net proliferation and invasion link biological aggressiveness assessed by MRI with hypoxia assessed by FMISO-PET in newly diagnosed glioblastomas. Cancer Res 69: 4502-4509, 2009.

33. Neal ML, Trister AD, Cloke T, Sodt R, Ahn S, et al: Discriminating survival outcomes in patients with glioblastoma using a simulation-based, patient-specific response metric. PLoS One 8: e51951, 2013

34. Demuth T and Berens ME: Molecular mechanisms of glioma cell migration and invasion. J Neurooncol 70: 217-228, 2004. 